The Free Internet Journal

for Organic Chemistry
Paper

Arkivoc 2018, part iv, 70-75

\title{
Indole anion cycloadditions with methyl coumalate
}

\author{
George A. Kraus* and Huangchao Yu \\ Department of Chemistry, lowa State University, Ames, IA 50011 \\ Email:gakraus@iastate.edu
}

\section{Dedication}

We honor Professor Gordon Gribble on his distinguished career in organic chemistry. His legacy of versatile synthetic methodology and total synthesis will continue to be valued by both academic and industrial researchers

Received 01-02-2018

Accepted 01-27-2018

Published on line 02-27-2018

\section{Abstract}

The reaction of the anion of indoles with methyl coumalate followed by selective opening of the lactone led to the preparation of tricyclic intermediates for indole alkaloid synthesis.<smiles>COC(=O)c1ccc(=O)oc1</smiles><smiles></smiles>

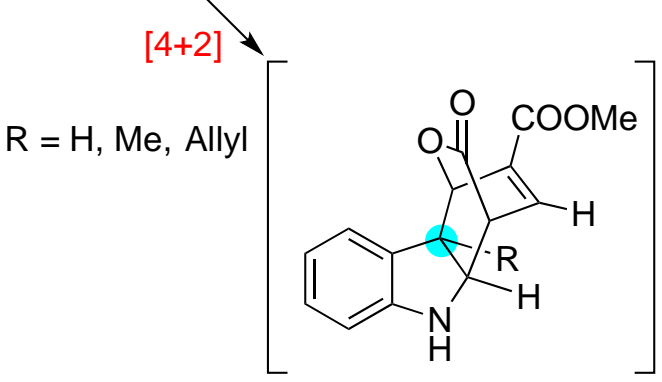

separable intermediate
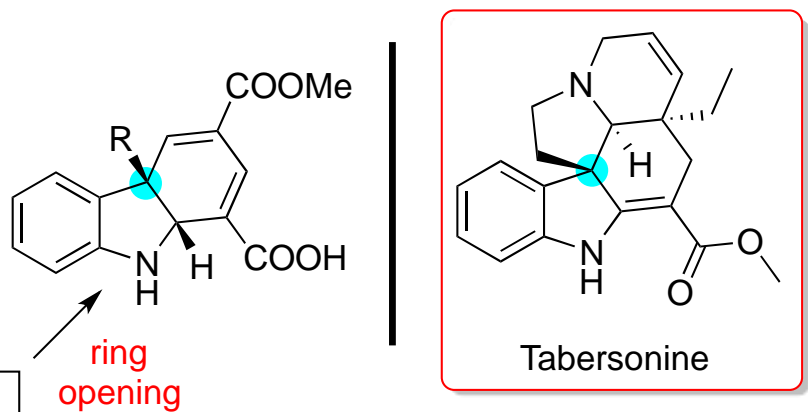

Keywords: Pyrone, indole, cycloaddition, anion 


\section{Introduction}

The construction of indoles remains an active area in synthetic and medicinal chemistry. The incredible diversity among indole-containing structures inspires new synthetic strategies that often require new methodology. Recent synthetic approaches to polycyclic indoles include the innovative strategies of Stoltz ${ }^{1}$ and Sarpong. ${ }^{2}$ The Gribble research group and others laid the groundwork for many of these achievements. His innovative studies on nucleophilic and radical additions to indoles and his use of furo[3,4- $b$ ] indoles in synthesis have had an impact on the field. . $^{3,4,5,6}$ Additionally, his reviews on indole chemistry have been widely referenced. ${ }^{7}$

We recently reported the preparation of substituted carbazoles from pyrones via the reaction of 3chloroindoles and methyl coumalate 1 at $200{ }^{\circ} \mathrm{C}$ as shown in Scheme $1 .{ }^{8}$ This reaction involves an inverse electron demand Diels-Alder reaction followed by elimination of hydrogen chloride and carbon dioxide. A recent computational chemistry study of this reaction showed that the elimination of $\mathrm{HCl}$ preceded the elimination of carbon dioxide. ${ }^{9}$<smiles>COC(=O)c1ccc(=O)oc1</smiles>

1<smiles>Clc1cn(Cc2ccccc2)c2ccccc12</smiles>

$\mathrm{Bn}$<smiles>CC(=O)c1ccc2c(c1)c1ccccc1n2Cc1ccccc1</smiles>

Scheme 1. Diels-Alder reaction of 3-chloroindole and methyl coumalate.

In order to retain the lactone, we needed to accomplish this connection at much lower temperatures. We investigated the reaction of the anion of indole with methyl coumalate at subambient temperature. Annulations initiated by indole anions are not common. Bäckvall and coworkers reported an interesting cyclization of 2-sulfonyl dienes with the anion of indole at $0{ }^{\circ} \mathrm{C} .{ }^{10}$ They obtained a tetrahydrocarbazole in good yield which they termed a sequential Michael addition reaction (Scheme 2). In a related approach, Marko showed that the anion of indole reacted with certain vinyl acrylates to generate useful yields of tetrahydrocarbazoles. ${ }^{11}$

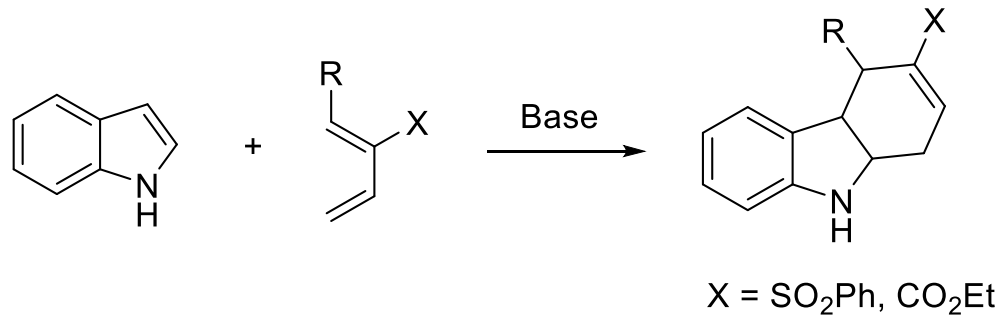

Scheme 2. Diels-Alder reaction of the indole anion and substituted dienes. 


\section{Results and Discussion}

The reaction of coumalate 1 with the magnesium salt of indole (generated in situ at $0{ }^{\circ} \mathrm{C}$ with isopropylmagnesium chloride) in THF afforded tetracyclic lactone adduct as a mixture of isomers, which underwent a retro-Diels-Alder process at room temperature. Other attempts reacting indolemagnesium chloride with methyl coumalate in THF resulted in a mixture of cycloaddition adducts along with a low yield of 3a. Fortunately, changing the Grignard reagent to methylmagnesium bromide or methylmagnesium iodide gave a higher yield of $\mathbf{3 a}$. Our best result was using a 1:1 mixture of toluene/diethyl ether as solvent and methylmagnesium iodide as the base, affording lactone ring opened product dihydrocarbazole acid $\mathbf{3 a}$ in $75 \%$ yield, in one pot (Scheme 3). The structure was supported by new ${ }^{1} \mathrm{H}$ NMR resonances at $7.83 \mathrm{ppm}\left(\mathrm{H}_{\mathrm{a}}\right.$, singlet) and $7.61 \mathrm{ppm}\left(\mathrm{H}_{\mathrm{b}}\right.$, doublet, $\left.J=2.8 \mathrm{~Hz}\right)$.

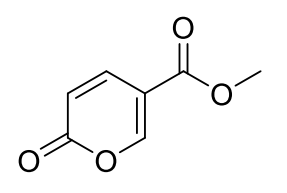

1<smiles>c1ccc2[nH]ccc2c1</smiles>

$2 \mathbf{a}$

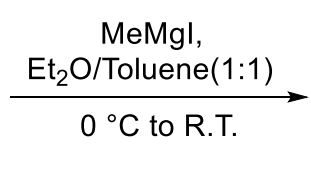

$0{ }^{\circ} \mathrm{C}$ to R.T.

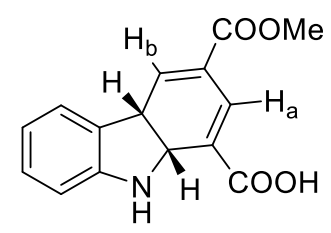

$3 a$

Scheme 3. Synthesis of dihydrocarbazole $3 a$.

To our surprise, the reaction of methyl coumalate $\mathbf{1}$ with 3 -methylindole $\mathbf{2} \mathbf{b}$ under the same conditions afforded the tetracyclic lactone $\mathbf{4}$ in $67 \%$ yield as a single diastereomer after recrystallization, which could also undergo a spontaneous retro-Diel-Alder process at room temperature. In an attempt to obtain the lactone ring-opened product $\mathbf{3 b}$, we treated the cyclic lactone $\mathbf{4}$ with hydrochloric acid in THF/water mixture, but the ring opened product was not observed. Then 4 was treated with trifluoroacetic acid in THF, but no product was observed at $-20{ }^{\circ} \mathrm{C}$. Eventually, we found that 4 could be converted into dihydrocarbazole acid $\mathbf{3 b}$ by reaction with dilute trifluoromethanesulfonic acid at $-20{ }^{\circ} \mathrm{C}$ in $29 \%$ yield (Scheme 4 ). The endo-structure of 4 was assigned by NOE experiments: an ${ }^{1} \mathrm{H}-\mathrm{NOE}$ effect was observed between the methyl of the ester and the vinyl hydrogen, as well as between the quaternary methyl group and the vinyl hydrogen.

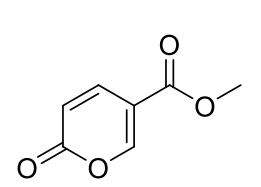

1

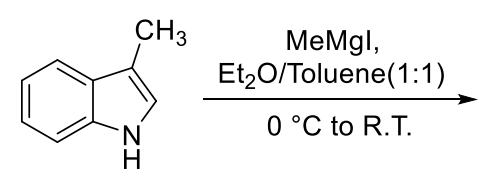

$\mathbf{2 b}$

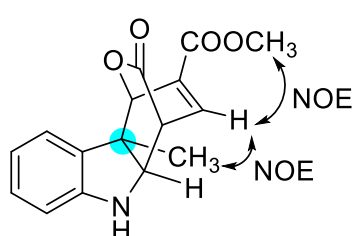

4

$\mid \begin{gathered}\text { TfOH, THF, } \\ 7 \text { days, }-20^{\circ} \mathrm{C}\end{gathered}$

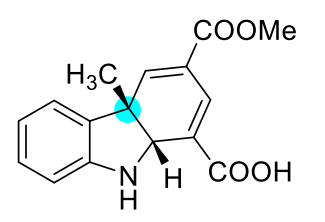

3b

Scheme 4. Synthesis of $\mathbf{3 b}$. 
Finally, reaction of 3-allyl indole $\mathbf{2 c}$ (produced from the palladium-catalyzed allylation of indole by the method of Larionov ${ }^{12}$ ) with methyl coumalate gave lactone adducts as a 2:3 mixture of diastereomers (exo and endo) in $43 \%$ yield, which could be stored at ambient temperature for a shot time. Acid-catalyzed ring opening gave $3 c$ in $31 \%$ yield (Scheme 5 ).

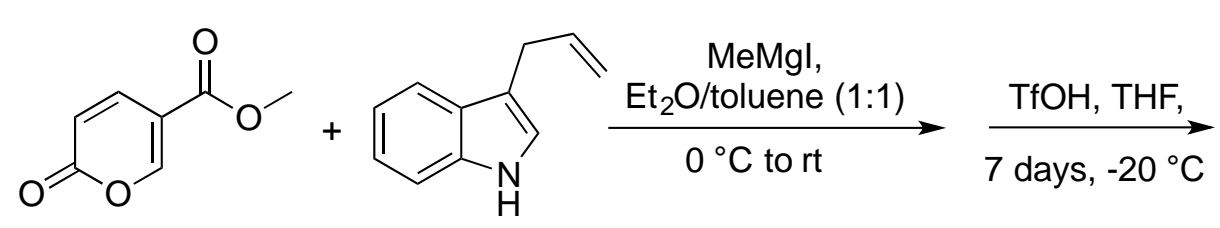

1

2c<smiles>C=CCC1C=C(C(C)=O)C=C(C(=O)O)C1Cc1ccccc1</smiles>

3c

Scheme 5. Synthesis of alkaloid precursor 3c.

Numerous natural products have been reported containing structures with a fused hydrocarbazole ring system, such as strychnine and tabersonine (Scheme 6). ${ }^{13,14}$ The allyl group in $\mathbf{3 c}$ could be potentially transformed to other functional groups.

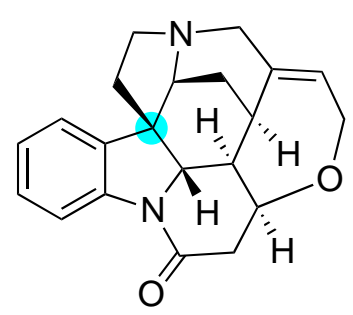

strychnine

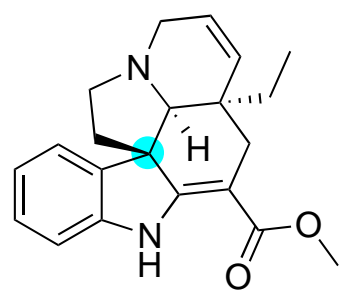

tabersonine

Scheme 6. Natural products with fused hydrocarbazole structure.

\section{Conclusions}

We have successfully developed a method for construction of dihydrocarbazoles in a two-step transformation from commercially available materials. Construction of a quaternary center at the 3-position of the indole ring could also be achieved in moderate yield. This methodology could be applied to the synthesis of complex indoles.

\section{Experimental Section}

General. All starting materials were purchased from Sigma-Aldrich and AK Scientific Institution. Solvents were purchased from Sigma-Aldrich and Fisher Scientific, and used without further purification. All reactions were monitored by thin layer chromatography (TLC). All yields refer to separated yields after column chromatography. TLC was carried out on silica plates using UV light as a visualizing agent and 
phosphomolybdic acid (PMA) or potassium permanganate solution with heat. All columns were performed with silica gel $60 \AA$, particle size $40-63 \mu \mathrm{m} .{ }^{1} \mathrm{H}$ and ${ }^{13} \mathrm{C} N M R$ spectra were acquired in deuterated solvents on a Varian MR-400 or Bruker Avance III 600 MHz spectrometer.

3-(Methoxycarbonyl)-4a,9a-dihydro-9H-carbazole-1-carboxylic acid (3a). Under an argon atmosphere, indole (176 mg, $1.5 \mathrm{mmol}, 1.5$ equiv.) was dissolved in dry toluene $(3 \mathrm{~mL})$ and dry $\mathrm{Et}_{2} \mathrm{O}(3.5 \mathrm{~mL})$ at $0{ }^{\circ} \mathrm{C}$, then methylmagnesium iodide solution ( $3.0 \mathrm{M}$ in $\mathrm{Et}_{2} \mathrm{O}, 0.43 \mathrm{~mL}, 1.3$ equiv) was added dropwise. The reaction mixture was stirred at $0{ }^{\circ} \mathrm{C}$ for 15 minutes to fully deprotonate, and then a suspension of methyl coumalate (154 mg, $1.0 \mathrm{mmol}, 1.0$ equiv) in dry toluene $(1.0 \mathrm{~mL})$ was added in one portion. Then the mixture was sonicated until a yellow suspension formed (about 1 minute). After an additional 5 min stirring at rt, the reaction was quenched by adding saturated aq ammonium chloride solution, and was extracted with EtOAc (3x), and was dried over $\mathrm{Na}_{2} \mathrm{SO}_{4}$. Recrystallization from $\mathrm{CH}_{2} \mathrm{Cl}_{2}$ /hexane gave the product as yellow crystals (203 $\mathrm{mg}, 75 \%$ yield); mp 163-165 ${ }^{\circ} \mathrm{C} ;{ }^{1} \mathrm{H}$ NMR (400 MHz, DMSO-d $\mathrm{d}_{6} \delta 12.31(\mathrm{~s}, 1 \mathrm{H}), 11.93(\mathrm{~s}, 1 \mathrm{H}), 7.83(\mathrm{~s}, 1 \mathrm{H}), 7.77$ $(\mathrm{d}, J 7.8,1 \mathrm{H}), 7.61(\mathrm{~d}, J 2.8,1 \mathrm{H}), 7.48(\mathrm{~d}, J$ 8.0, 1H), $7.22(\mathrm{t}, J \mathrm{~J} .3,1 \mathrm{H}), 7.16(\mathrm{t}, J 7.3,1 \mathrm{H}), 6.90(\mathrm{~d}, J 11.6,1 \mathrm{H}), 6.04$ (d, J 11.5, 1H), 3.65 (s, 3H); ${ }^{13} \mathrm{C}$ NMR (101 MHz, DMSO-d $) \delta$ 167.2, 167.1, 138.0, 136.0, 131.5, 129.5, 127.0, 123.5, 123.0, 122.6, 120.6, 118.2, 112.2, 110.9, 51.4; HRMS (ESI-QTOF) calcd for $\mathrm{C}_{15} \mathrm{H}_{13} \mathrm{NO}_{4}[\mathrm{M}+\mathrm{H}]^{+} 272.0917$, found 272.0910 .

Methyl 4a-methyl-11-oxo-4,4a,9,9a-tetrahydro-1H-4,1-(epoxymethano)carbazole-3-carboxylate (4). Using the same procedure as for $3 a$, with 3-methylindole $(787 \mathrm{mg}, 6.0 \mathrm{mmol}, 1.2$ equiv), methyl coumalate (771 mg, $5.0 \mathrm{mmol}, 1.0$ equiv.) and methylmagnesium iodide solution (3.0 $\mathrm{M}$ in $\mathrm{Et}_{2} \mathrm{O}, 1.83 \mathrm{~mL}, 1.1$ equiv). The final product 4 was recrystallized from EtOAc/hexane at $-20{ }^{\circ} \mathrm{C}$ as light yellow crystals (950 mg, $67 \%$ yield), which can be stored at $-20{ }^{\circ} \mathrm{C}$ for weeks (decomposed to starting materials at room temperature). ${ }^{1} \mathrm{H} \mathrm{NMR}(400 \mathrm{MHz}$, DMSO- $\left.d_{6}\right) \delta 12.44(\mathrm{~s}, 1 \mathrm{H}), 8.13(\mathrm{~s}, 1 \mathrm{H}), 7.71(\mathrm{~d}, J$ 8.1, $1 \mathrm{H}), 7.58(\mathrm{~d}, J 7.7,1 \mathrm{H}), 7.45(\mathrm{~d}, J 1.5,1 \mathrm{H}), 7.32(\mathrm{t}, J 7.4$, $1 \mathrm{H}), 7.25(\mathrm{t}, J \mathrm{~T} .6,1 \mathrm{H}), 6.94(\mathrm{dd}, J 11.5,1.6,1 \mathrm{H}), 6.13(\mathrm{~d}, J 11.5,1 \mathrm{H}), 3.68(\mathrm{~s}, 3 \mathrm{H}), 2.27(\mathrm{~s}, 3 \mathrm{H}) ;{ }^{13} \mathrm{C} \mathrm{NMR}(101$ $\left.\mathrm{MHz}, \mathrm{DMSO}-d_{6}\right) \delta 166.7,166.5,136.8,135.7,132.3,129.3,125.1,123.8,123.7,122.2,119.2,117.2,111.4$, 110.6, 51.7, 9.4; HRMS (ESI-QTOF) calcd for $\mathrm{C}_{16} \mathrm{H}_{15} \mathrm{NO}_{4}[\mathrm{M}+\mathrm{H}]^{+} 286.1074$, found 286.1073.

3-(Methoxycarbonyl)-4a-methyl-4a,9a-dihydro-9H-carbazole-1-carboxylic acid (3b). Compound 4 (110 mg, $0.34 \mathrm{mmol}$ ) was dissolved in dry THF at rt. To it, 3 drops of trifluoromethanesulfonic acid were added, and the solution was stored at $-20^{\circ} \mathrm{C}$ for 7 days. After the reaction was completed, the mixture was dissolved in EtOAc, and washed three times with $\mathrm{H}_{2} \mathrm{O}$, then with brine. The organic phase was dried over $\mathrm{Na}_{2} \mathrm{SO}_{4}$. Recrystallization from $\mathrm{CH}_{2} \mathrm{Cl}_{2}$ /hexane gave the product $3 \mathrm{~b}$ as a yellow solid (32 mg, 29\% yield). $\mathrm{mp} 179-180{ }^{\circ} \mathrm{C} ;{ }^{1} \mathrm{H} \mathrm{NMR}(600$ $\left.\mathrm{MHz}, \mathrm{DMSO}-\mathrm{d}_{6}\right) \delta 12.46(\mathrm{~s}, 1 \mathrm{H}), 8.38(\mathrm{~s}, 1 \mathrm{H}), 7.71(\mathrm{~d}, J$ 8.2, $1 \mathrm{H}), 7.64(\mathrm{dt}, J \mathrm{~J} .7,0.9,1 \mathrm{H}), 7.55(\mathrm{dt}, J 15.8,1.0,1 \mathrm{H})$, $7.41(\mathrm{~d}, J 1.4,1 \mathrm{H}), 7.37(\mathrm{td}, J$ 7.1, 1.3, 1H), 7.31 (td, J 7.5, 0.9, 1H), $6.52(\mathrm{~d}, J 15.7,1 \mathrm{H}), 3.83(\mathrm{~s}, 3 \mathrm{H}), 2.32(\mathrm{~d}, J$ 1.2, 3H); ${ }^{13} \mathrm{C}$ NMR (151 MHz, DMSO- $\left.d_{6}\right) \delta 167.7,166.1,137.1,136.9,135.4,129.7,124.3,124.1,123.1,122.7$, 119.4, 118.4, 111.0, 110.3, 52.1, 9.5; HRMS (ESI-QTOF) calcd for $\mathrm{C}_{16} \mathrm{H}_{15} \mathrm{NO}_{4}[\mathrm{M}+\mathrm{H}]^{+}$286.1074, found 286.1079.

4a-Allyl-3-(methoxycarbonyl)-4a,9a-dihydro-9H-carbazole-1-carboxylic acid (3c). 3c was synthesized according to the previous procedure for $\mathbf{3 b}$ to give yellow crystals ( $31 \%$ yield) after recrystallization in $\mathrm{CH}_{2} \mathrm{Cl}_{2}$ /hexane. $\mathrm{mp} 73-75{ }^{\circ} \mathrm{C} ;{ }^{1} \mathrm{H}$ NMR $\left(400 \mathrm{MHz}\right.$, acetone- $\left.d_{6}\right) \delta 8.44(\mathrm{~s}, 1 \mathrm{H}), 7.74(\mathrm{dd}, J 15.8,0.8,1 \mathrm{H}), 7.70-$ $7.62(\mathrm{~m}, 2 \mathrm{H}), 7.43(\mathrm{~s}, 1 \mathrm{H}), 7.37(\mathrm{td}, J 7.1,1.2,1 \mathrm{H}), 7.29(\mathrm{td}, J 7.1,1.0,1 \mathrm{H}), 6.69(\mathrm{~d}, J 15.9,1 \mathrm{H}), 6.06(\mathrm{ddt}, J 16.7$, 10.0, 6.5, 1H), 5.21 (dq, J 17.1, 1.7, 1H), 5.09 (ddt, J 10.0, 2.0, 1.4, 1H), $3.86(\mathrm{~s}, 3 \mathrm{H}), 3.55(\mathrm{dt}, J 6.5,1.4,2 \mathrm{H}) ;{ }^{13} \mathrm{C}$ NMR $\left(101 \mathrm{MHz}\right.$, acetone- $\left.d_{6}\right) \delta 166.9,138.7,137.9,136.9,136.8,136.8,130.1,125.2,125.1,123.7,123.7$, 123.6, 122.3, 120.7, 116.6, 111.6, 52.3; HRMS (ESI-QTOF) calcd for $\mathrm{C}_{18} \mathrm{H}_{17} \mathrm{NO}_{4}[\mathrm{M}+\mathrm{H}]^{+} 312.1230$, found 312.1222. 


\section{Acknowledgements}

We thank the NSF Engineering Center for Biorenewable Chemicals (CBiRC) which was awarded NSF grant EEC0813570 for support of this research.

\section{References}

1. Pritchett, B. P.; Donckele, E. J.; Stoltz, B. M. Angew. Chem. Int. Ed. 2017, 56, 12624. https://doi.org/10.1002/anie.201707304

2. Mukai, K.; de Sant'Ana, D. P.; Hirooka, Y.; Sarpong, R. et al. Nat. Chem. 2017, Ahead of Print., https://doi.org/10.1038/nchem.2862

3. Lopchuk, J. M.; Montgomery, W. L.; Jasinski, J. P.; Gorjifard, S.; Gribble, G. W. Tetrahedron Lett. 2013, 54, 6142.

https://doi.org/10.1016/j.tetlet.2013.09.012

4. Qian, D. C.; Alford, P. E.; Kishbaugh, T. L. S.; Jones, S. T.; Gribble, G. W. Arkivoc 2010, (4), 66. https://doi.org/10.3998/ark.5550190.0011.406

5. Moskalev, N. V.; Gribble, G. W. Tetrahedron Lett. 2002, 43, 197.

https://doi.org/10.1016/S0040-4039(01)02068-8

6. Liu, Y.; Gribble, G. W. Tetrahedron Lett. 2002, 43, 7135.

https://doi.org/10.1016/S0040-4039(02)01710-0

7. Gribble, G. W. J. Chem. Soc., Perkin Trans. 1 2000, 1045.

https://doi.org/10.1039/A909834H

8. Guney, T.; Lee, J. J.; Kraus, G. A. Org. Lett. 2014, 16, 1124.

https://doi.org/10.1021/ol403733n

9. Domingo, L. R.; Saez, J. A.; Emamian, S. R. Org. Biomol. Chem. 2015, 13, 2034.

https://doi.org/10.1039/C4OB02340D

10. Bäckvall, J. E.; Plobeck, N. A.; Juntunen, S. K. Tetrahedron Lett. 1989, 30, 2589. https://doi.org/10.1016/S0040-4039(01)80458-5

11. Marko, I. E.; Chesney, A. Synlett, 1992, 275. https://doi.org/10.1055/s-1992-21337

12. Zhang, Y., Stephens, D., Hernandez, G., Mendoza, R. and Larionov, O. V. Chem. Eur. J. 2012, $18,16612$. https://doi.org/10.1002/chem.201203435

13. O'Connor, S. E.; Maresh, J. J. Nat. Prod. Rep. 2006, 23, 532.

https://doi.org/10.1039/B512615K

14. Qu, Y.; Simonescu, R.; De Luca, V. J. Nat. Prod., 2016, 79, 3143

https://doi.org/10.1021/acs.jnatprod.6b00405 